\title{
E Perioperative outcomes and adverse events of minimally invasive surgery during transforaminal lumbar interbody fusion/posterior lumbar interbody fusion
}

\author{
Robert F. Heary, MD,' and Michael G. Kaiser, MD² \\ 'Department of Neurosurgery, Rutgers New Jersey Medical School, Newark, New Jersey; and 2Neurological Surgery, Columbia \\ University, New York, New York
}

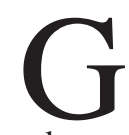
OLDSTEIN et al. performed a systematic review of the literature and a meta-analysis of the study results comparing acute perioperative outcomes and adverse event rates of minimally invasive surgery (MIS) versus open transforaminal lumbar interbody fusion (TLIF)/posterior lumbar interbody fusion (PLIF) surgery for degenerative lumbar disease. ${ }^{1}$ The authors also summarized data pertaining to patient-reported outcomes, fusion status, and reoperation rates. From 3301 papers, 26 studies met the inclusion criteria for this analysis. Only 1 prospective randomized controlled trial (RCT) was included, whereas the remaining 25 were either prospective or retrospective comparative studies.

The authors have conducted a thorough and exhaustive review of the literature and are to be congratulated for incorporating measures outlined in the Meta-analysis of Observational Studies in Epidemiology (MOOSE) and Preferred Reporting Items for Systematic Reviews and Meta-analyses (PRISMA) guidelines for the performance and reporting of systematic reviews and meta-analyses. Utilizing the Grades of Recommendation, Assessment, Development, and Evaluation (GRADE) protocol, the strength of the included studies was designated as "low" and "very low" quality. By pooling populations among these studies, data from more than 800 patients in each treatment cohort were analyzed. Although no significant difference in operative time was identified, estimated blood loss, time to ambulation, and length of hospital stay all favored MIS techniques. Adverse event rates were similar between the two groups; however, the incidence of medical complications was higher in the open cohort. No differences in the rates of nonunion or reoperation were identified. Both patient populations demonstrated improved outcomes; however, a small, but significantly superior, improvement in ODI scores was observed following MIS procedures.

As with any literature review, this study is limited by the strength and quality of the data analyzed. Compared to other reviews, this investigation benefits from the inclusion of only comparative studies and the thorough analysis of methodological quality. Heterogeneity among patients, small sample sizes, lack of consistent reporting, and subjective treatment allocation are among the major limitations that introduce bias in these studies reviewed. Without well-designed RCTs, it is possible that an applesto-oranges situation exists wherein the less difficult cases would be treated with MIS procedures. This could certainly impact patient outcomes and compromise the validity of any analysis. In addition, the definition of MIS techniques is often ambiguous in many of these studies.

A potential issue is that authors are writing articles comparing the newer MIS techniques versus the older open procedures, and as such, there may be a bias towards demonstrating more favorable outcomes for the newer MIS procedures. Although not clearly identified in this review, major concerns regarding studies analyzing MIS procedures have been the duration of follow-up and method, or lack of, fusion assessment.

Despite substantial limitations in the available literature on this topic, the authors have done an excellent job of summarizing the available data and providing a thoughtful discussion. Their conclusion, that a well-designed, 
multicenter RCT utilizing validated outcomes measures is needed to perform a valid comparison of these two approaches, is well supported.

http://thejns.org/doi/abs/10.3171/2015.3.SPINE15238

\section{Reference}

1. Goldstein CL, Macwan K, Sundararajan K, Rampersaud YR: Perioperative outcomes and adverse events of minimally invasive versus open posterior lumbar fusion: meta-analysis and systematic review. J Neurosurg Spine [epub ahead of print November 13, 2015. DOI: 10.3171/2015.2.SPINE14973]

\section{Disclosure}

The authors report no conflict of interest.

\section{Response}

Christina L. Goldstein, MD, FRCSC, ${ }^{1}$ and Y. Raja Rampersaud, MD, FRCSC ${ }^{2}$

${ }^{1}$ Missouri Orthopaedic Institute, University of Missouri, Columbia, Missouri; and 2Department of Surgery, Division of Orthopaedics, University of Toronto, Ontario, Canada

Thank you for the opportunity to respond to this issue's editorial by Dr. Heary and Dr. Kaiser regarding our submission "Perioperative outcomes and adverse events of minimally invasive versus open posterior lumbar fusion: meta-analysis and systematic review."

While very complimentary of our study methodology, Drs. Heary and Kaiser appropriately point out that the quality of the results and ability to draw conclusions in systematic reviews and meta-analyses are limited by the quality of the included studies. Our adherence to MOOSE and PRISMA guidelines and transparent reporting of the results of our GRADE quality assessment of the 26 studies included in our review in no way eradicates this limitation; instead, it simply functions to educate the reader and facilitate reproduction of our results.

Regarding the concerns raised about comparing new MIS techniques to older open procedures, the majority of the included studies compared concurrent open and MIS cohorts. Three studies failed to report the time period of data collection for either of their treatment groups. ${ }^{3,10,11}$ Scheufler et al. ${ }^{9}$ reported the inclusion period of their MIS cohort, but did not report whether the open cohort was historical or concurrent, and Tsutsumimoto et al. ${ }^{12}$ compared their MIS group to a historical open cohort. Although it is possible that some differences in care may have changed over time, which could have impacted the observed results, these studies represent only $16.6 \%$ of the open $(\mathrm{n}=$ $134)$ and $15.0 \%$ of the MIS $(n=138)$ cases. We therefore believe the impact of differences in treatment time periods on our study results would be minimal.

Heary and Kaiser also correctly point out that differences in duration of follow-up may impact our study results. Two of the included 26 papers failed to report their follow-up period, ${ }^{4,15}$ and 6 others reported differential follow-up times for the open and MIS cohorts. , $, 2,5,6,7,10_{\text {How- }}$ ever, the ranges of follow-up for the open and MIS cohorts in these 6 studies were fairly similar at 13.5 to 40 months and 14 to 32 months, respectively. The slightly longer follow-up duration in the open cohorts is certainly another potential source of bias; however, this can be bidirectional and differentially favor either cohort. Overall, given the clinically insignificant differences in patient reported outcomes between open surgery and MIS, it is unlikely that more uniform follow-up would have altered our conclusion of equivalent patient-reported outcomes at a median of 2 years following surgery.

Finally, it is also true that variability in fusion assessment methods may have impacted our study results. In the 8 studies reporting on fusion rates, ${ }^{1,3,5,6-8,13,14} 1$ study failed to report their method of fusion assessment and the remaining 7 each used different methods of determining solid fusion. Although this introduces significant heterogeneity into the pooled nonunion rates presented in our meta-analysis, it should not impact the within-study comparisons given that for each comparative study the same method of fusion assessment was used in the open and MIS cohorts. Furthermore, fusion status may not impact the aggregate patient-reported outcomes for the duration of follow-up observed in the included studies.

Despite these and other limitations addressed in our paper, the greatest of which we believe is diagnostic heterogeneity, the results of our systematic review and metaanalysis represent the best available evidence regarding comparative outcomes of open versus minimally invasive techniques for transforaminal and posterior lumbar interbody fusion. Furthermore, our study serves to highlight that multicenter controlled studies, with appropriate diagnostic, clinical, and surgical stratification, are desperately required to definitively address the question of superiority of 1 technique over another.

\section{References}

1. Dhall SS, Wang MY, Mummaneni PV: Clinical and radiographic comparison of mini open transforaminal lumbar interbody fusion with open transforaminal lumbar interbody fusion in 42 patients with long-term follow-up. J Neurosurg Spine 9:560-565, 2008

2. Fan S, Hu Z, Zhao F, Zhao X, Huang Y, Fang X: Multifidus muscle changes and clinical effects of one-level posterior lumbar interbody fusion: minimally invasive procedure versus conventional open approach. Eur Spine J 19:316-324, 2010

3. Harris EB, Sayadipour A, Massey P, Duplantier NO, Anderson DG: Mini-open versus open decompression and fusion for lumbar degenerative spondylolisthesis with stenosis. Am J Orthop (Belle Meade NJ) 40:E257-E261, 2011

4. Isaacs RE, Podichetty VK, Santiago P, Sandhu FA, Spears J, Kelly K, et al: Minimally invasive microendoscopy-assisted transforaminal lumbar interbody fusion with instrumentation. J Neurosurg Spine 3:98-105, 2005

5. Kotani Y, Abumi K, Ito M, Sudo H, Abe Y, Minami A: Midterm clinical results of minimally invasive decompression and posterolateral fusion with percutaneous pedicle screws versus conventional approach for degenerative spondylolisthesis with spinal stenosis. Eur Spine J 21:1171-1177, 2012

6. Lau D, Lee JG, Han SJ, Lu DC, Chou D: Complications and perioperative factors associated with learning the technique of minimally invasive transforaminal lumbar interbody fusion (TLIF). J Clin Neurosci 18:624-627, 2011

7. Mobbs RJ, Sivabalan P, Li J: Minimally invasive surgery 
compared to open spinal fusion for the treatment of degenerative lumbar spine pathologies. J Clin Neurosci 19:829-835, 2012

8. Park Y, Ha JW: Comparison of one-level posterior lumbar interbody fusion performed with a minimally invasive approach or a traditional open approach. Spine (Phila Pa 1976) 32:537-543, 2007

9. Scheufler KM, Dohmen H, Vougioukas VI: Percutaneous transforaminal lumbar interbody fusion for the treatment of degenerative lumbar instability. Neurosurgery 60:203-213, 2007

10. Schizas C, Tzinieris N, Tsiridis E, Kosmopoulos V: Minimally invasive versus open transforaminal lumbar interbody fusion: evaluating initial experience. Int Orthop 33:16831688,2009

11. Starkweather AR, Witek-Janusek L, Nockels RP, Peterson J, Mathews HL: The multiple benefits of minimally invasive spinal surgery: results comparing transforaminal lumbar interbody fusion and posterior lumbar fusion. J Neurosci Nurs 40:32-39, 2008
12. Tsutsumimoto T, Shomogata M, Ohta H, Misawa H: Miniopen versus conventional open posterior lumbar interbody fusion for the treatment of lumbar degenerative spondylolisthesis: comparison of paraspinal muscle damage and slip reduction. Spine (Phila Pa 1976) 34:1923-1928, 2009

13. Wang J, Zhou Y, Zhang ZF, Li CQ, Zheng WJ, Liu J: Comparison of the clinical outcome in overweight or obese patients after minimally invasive versus open transforaminal lumbar interbody fusion. J Spinal Disord Tech 27:202-206, 2014

14. Wang J, Zhou Y, Zhang ZF, Li CQ, Zheng WJ, Liu J: Minimally invasive or open transforaminal lumbar interbody fusion as revision surgery for patients previously treated by open discectomy and decompression of the lumbar spine. Eur Spine J 20:623-628, 2011

15. Wang MY, Cummock MD, Yu Y, Trivedi RA: An analysis of the differences in the acute hospitalization charges following minimally invasive versus open posterior lumbar interbody fusion. J Neurosurg Spine 12:694-699, 2010 